\section{Three patterns of chronic cerebrospinal venous insufficiency in Ménière syndrome patients: Diagnosis and treatment options}

\author{
Aldo Messina, ${ }^{1}$ Girolamo Garofalo, ${ }^{1}$ \\ Antonella Faletra, ${ }^{1}$ Davide Piraino ${ }^{2}$ \\ ${ }^{1}$ Audiology Section; ${ }^{2}$ Interventional \\ Cardiology Unit, University Hospital P. \\ Giaccone, Palermo, Italy
}

\begin{abstract}
Identification techniques of the three different chronic cerebrospinal venous insufficiency patterns and related treatment options are in an initial phase of evaluation and analysis. Our purpose is to describe the appropriate management, proposing a tailored approach for each one. We identified three different Ménière syndrome patients in our Audiology Department, diagnosing the corresponding chronic cerebrospinal venous insufficiency pattern by Echo-color Doppler ultrasound evaluation and treating by venous angioplasty or rehabilitative treatment according to the internal jugular and vertebral veins anomalies found in each patient. According to the pattern, after specific treatment, echo-color-Doppler control analysis revealed a normalized venous outflow correlated to Ménière symptoms reduction and/or progressive disappearance during one year follow up. An adequate analysis of venous cerebral and ear outflow and a tailored treatment may represent an effective option when chronic cerebrospinal venous insufficiency is correctly diagnosed.
\end{abstract}

\section{Introduction}

Cerebrospinal nervous system drainage was initially analyzed by Zamboni, identifying the possible anomalies of extracranial venous system responsible of chronic cerebrospinal venous insufficiency (CCSVI), ${ }^{1}$ classified among truncular venous malformations (TVM). ${ }^{2}$ These malformations occur during fetal life, causing by an altered development of the vascular trunk, with the consequence of possible venous anomalies as hypo or hyperplasia of the venous system, as well as intraluminal defects as fibrosis, septum and/or incomplete valve.

The innovation of these studies and the following literature of the last years is the fact that, before them, venous anomalies were considered as simple and irrelevant anatomical variants, without a clinical significance, because of: i) limited anatomical and physiological knowledge; ii) extreme interpersonal variability; iii) lack of codified parameters that could allow to identify and classify these venous anomalies.

The consequence was that the limited and never deepened understanding of the anatomy variants and pathophysiology mechanisms of extracranial venous system may consequently underestimate the impact and importance of cerebral and ear venous drainage abnormalities as well as their role in a variety of central nervous and ear system disorders. However CCSVI is currently well know, identifying three different patterns, according to the presence of vascular defects and/or extrinsic obstacle as muscle compression, ${ }^{3}$ that we have identified in three distinct patients afferent to the Audiology Department of our hospital.

Ethics board approval and patient publication consent form was obtained for this case series.

\section{Case \#1}

A 65-years-old woman was admitted to our Audiology Department for progressive hearing loss, (mainly in the right ear) associated to continuous and fastidious tinnitus and dizziness episodes with increased incidence in the last 15 year (weekly basis). Previous medical treatments used did not give effective and durable benefits to her condition. Cerebral computed tomography and magnetic resonance (MR) imaging scans were negative, while an audiometric exam confirmed a chronic right sensorineural hearing loss. Cerebrospinal outflow Echo-color Doppler (ECD) ultrasound evaluation of vertebral veins (VV) and internal jugular veins (IJV) both in supine position and upright was performed to assess the hemodynamic criteria of CCSVI, revealing a complete septum in the junction of right IJV and subclavian vein (the first part of J1 segment) with consequent outflow acceleration (Figure 1A), while left IJV revealed an hypoplasia in all the three assessed segments. These findings were confirmed by selective venography of both IJVs (Figure 1B), while analysis of Azygos vein (AV) reveled the absence of anomalies, so we proceeded to a balloon dilation of right IJV (16-mm-diameter and 40-mmlong balloon for $\mathrm{J} 1$ right segment) using a MAXI LD dilation catheter (Cordis, Johnson \& Johnson, Miami, Florida). Final venography (Figure 1C) and one month ECD analysis confirmed the improvement
Correspondence: Davide Piraino, Interventional Cardiology Unit and Audiology Section, University Hospital P. Giaccone, via del Vespro 128, Palermo, Italy.

Tel./Fax: +39.091.6554312.

E-mail: pirainod@libero.it

Key words: Chronic cerebrospinal venous insufficiency; Ménière syndrome.

Conflict of interest: the authors declare no potential conflict of interest.

Received for publication: 13 December 2019:

Revision received: 1 March 2020.

Accepted for publication: 25 March 2020.

This work is licensed under a Creative Commons Attribution 4.0 License (by-nc 4.0).

COCopyright: the Author(s), 2020

Licensee PAGEPress, Italy

Veins and Lymphatics 2020; 9:8758

doi:10.4081/vl.2020.8758

of cerebral outflow; moreover a progressive resolution of tinnitus and dizziness episodes as well as an audiometric examination improvement during one year follow up (Figure 1D).

\section{Case \#2}

A 54-years-old woman with fastidious and continuous bilateral tinnitus associated to auricular fullness (mainly in the left ear) and monthly episodes of dizziness related sometimes to autonomic phenomena (nausea and vomiting), was subjected to an audiometric examination (quite normal) as well as cerebral computed tomography and MR imaging scans (negative).

Cerebrospinal outflow ECD ultrasound evaluation of the VV and IJV both in supine position and upright revealed a partial muscular compression of right IJV and a complete muscular compression of left IJV in J2 and J3 segments (Figure 2A) in both positions, in almost complete resolution during Valsalva maneuver, revealing the real caliber of IJV and the absence of vascular anomalies with a normal outflow, denoting a type 2 of CCSVI.

We decided to direct the patient to a rehabilitative treatment for muscular compression of sternocleidomastoid (SCM) and omohyoid (OM) muscles, with the goal of a complete muscle relaxation and a restoration of physiological IJV caliber and venous flow. After a complete cycle of rehabilitative treatment, for the first time used for this CCSVI type by our team, ${ }^{4}$ we repeated 
ECD analysis observing a significant basal reduction of muscle compression and a normalized outflow through IJVs (Figure 2B) with a feeling of comfort for the patient due to tinnitus progressive attenuation and auricular fullness disappearance.

\section{Case \#3}

A 28-years-old-woman with a Ménière syndrome (MéS) diagnosed three years prior (auricular fullness associated to tinnitus and frequent dizziness episodes, sometimes on a daily basis) was admitted to our Audiology Department. Audiometric examination was normal and routine exam of cerebral computed tomography and magnetic resonance imaging scans were negative. Cerebrospinal outflow ECD ultra- sound evaluation in both supine position and upright revealed a significant muscle compression of both IJV in J2 and J3 segments (Figure 3A) and vascular anomalies described as criteria 1, 3 and 4 (Figure 3B) according to Zamboni classification. ${ }^{1}$ The IJV showed an annular constriction in $\mathrm{J} 1$ due to a rigid and immobile septum, so the association of muscular and vascular anomalies identified a type III CCSVI. After a complete cycle of rehabilitative treatment for sternocleidomastoid and omohyoid muscles relaxation, confirmed by ECD analysis that revealed a normalized $\mathrm{J} 2$ and J3 caliber and disappearance of muscle compression, we performed a selective venography of IJV (Figure 3C) and a balloon dilation (14-mm-diameter and 40-mmlong balloon for both IJV treatment) using a MAXI LD dilation catheter. During the phlebography was investigated the AV too, but no anomalies were revealed. Final venography (Figure 3D) and one month ECD assessment confirmed the normalized cerebral outflow. All three patients received one year follow up ECD analysis, confirming the persistent normalized cerebral outflow through the VV and IJV.

\section{Discussion}

In recent years, many authors tried to answer the question coming from Zamboni's initial study about CCSVI, how these extra-cranial venous anomalies may have pathologic consequences in the brain and ear physiology and which role in the common etiopathogenetic mechanism of different pathologies: initially associated exclusively to Multiple sclerosis (MS) and
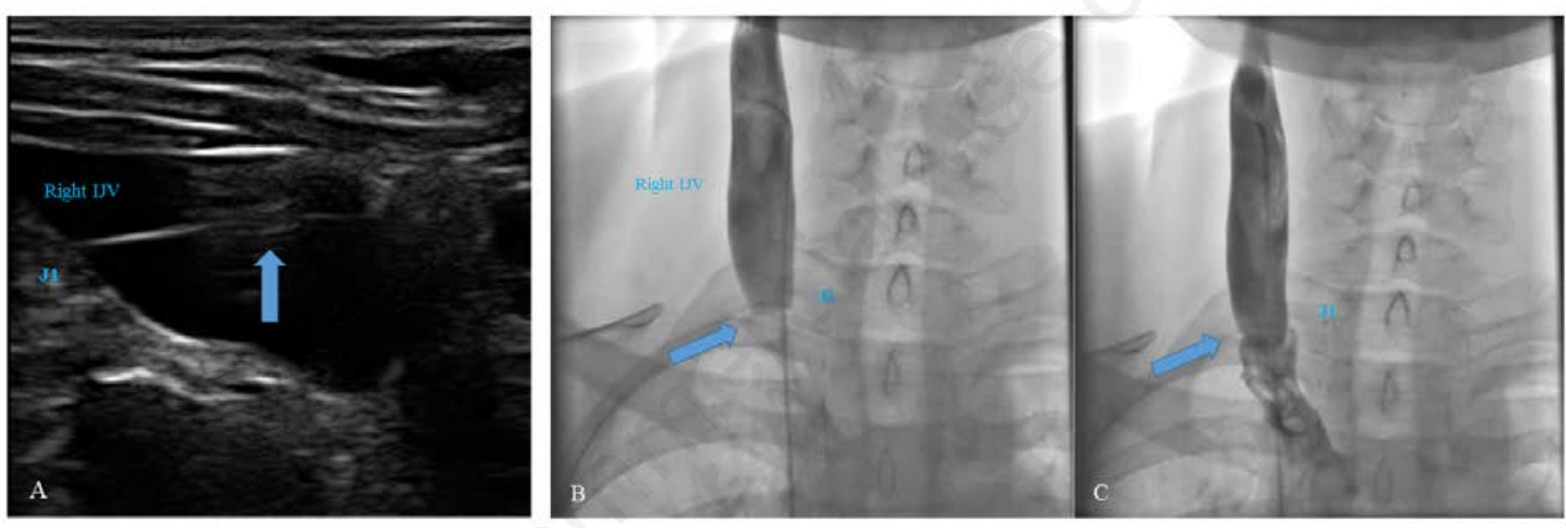

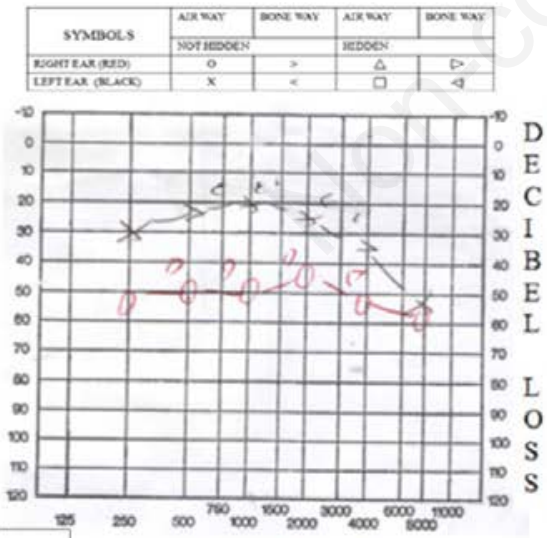

D

AUDIO FREQUENCY SCALE

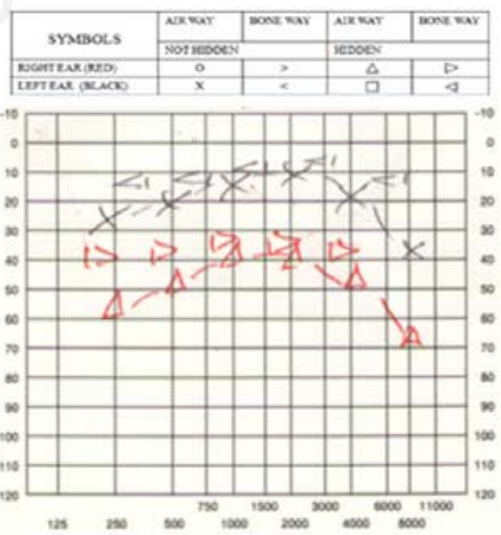

AUDIO FREQUENCY SCALE

Figure 1. Case 1: ECD analysis of VV and IJV (type 1 CCSVI, called Hydraulic). A) immobile septum in the terminal J1 segment of right IJV (blue arrow); B) selective venography of right IJV showing the immobile septum between J1 and succlavian vein (blue arrow) reducing considerably the caliber of the vessel and preventing the outflow of contrast medium to succlavian vein(the catheter overcomes this segment difficulty, occluding it completely); C) selective venography of right IJV after $16 / 40 \mathrm{~mm}$ balloon dilatation showing the resolution of vessel caliber constriction, removing the obstacle and allowing contrast medium outflow in succlavian vein (blue arrow); $D$ ) audiometry examination of the right ear (red signals) and left ear (black signals) via air and bone impulse transmission. In the left panel (before percutaneous transluminal angioplasty), pure-tone audiometry shows a low sensorineural hearing loss on the left side (20/30 $\mathrm{dB}$ ) and medium sensorineural hearing loss on the right side $(50 / 60 \mathrm{~dB})$. In the right panel (after percutaneous transluminal angioplasty), puretone audiometry shows bilateral low sensorineural hearing loss (improvement on the left and mainly on the right side). 
subsequently Parkinson or Alzheimer disease, MéS and migraine, ${ }^{5}$ as well as transient global amnesia, or retinal abnormalities $^{6}$ leading to the creation of a position paper with standardized noninvasive and invasive imaging protocols for evaluating extra-cranial venous abnormalities indicative of $\mathrm{CCSVI}^{7}$ by the use of ECD images to observe the vascular anomalies both structural and morphological as well as hemodynamic and when feasible the use of MR images with intravenous contrast agent allowing the evaluation of venous outflow abnormalities.

The key point is that these studies give venous system right dignity, because until now, it was been in the shadows of arterial system, in terms of considerations and insights in literature and clinical practice.

Moreover, the correct hunch was that, if cerebral and ear venous system is altered, it may have pathological impact as happen in other districts as lower limb, especially in a complicated district as head and neck.

The information and available data confirm that a correct ECD analysis by expert operators and an adequate treatment of extra-cranial venous anomalies may represent an effective option in many setting of patients, because of when CCSVI is present, in these different pathologies, it seems to participate to the appearance and/or increase lesions in the white matter and/or predisposing to the associated disorder and symptoms, altering the cerebrospinal fluid (CSF) dynamics and brain perfusion, as well as by promoting an excess of endolymphatic volume responsible of endolymphatic hydrops in Ménière patients ${ }^{8}$ with a specific and organ-related anomalies, because of there is a possible disease specific typology of abnormalities. ${ }^{9}$

Bavera et al. assessed as CCSVI may be the common mechanism in different pathologies as MS and MéS, demonstrating that not only there was a high prevalence of these anomalies in these two populations compared to a control population, but also as a predisposing factor that may promote them showing different patterns in the two pathologies. ${ }^{10}$

Their work underlines that neurological and ear pathologies so different as MS and MéS, may have a common element as CCSVI, taking a different form according to the pathology: anomalies mainly related to the $\mathrm{J} 1$ and $\mathrm{J} 2$ segments in MS patients, while J2-J3 segments anomalies in MéS, as well as jugular bulb anomalies and mediolateral or anteroposterior position that may determining encroachment of these structures and components.

Mandolesi et al. reported a classification of CCSVI in three different patterns: ${ }^{3}$

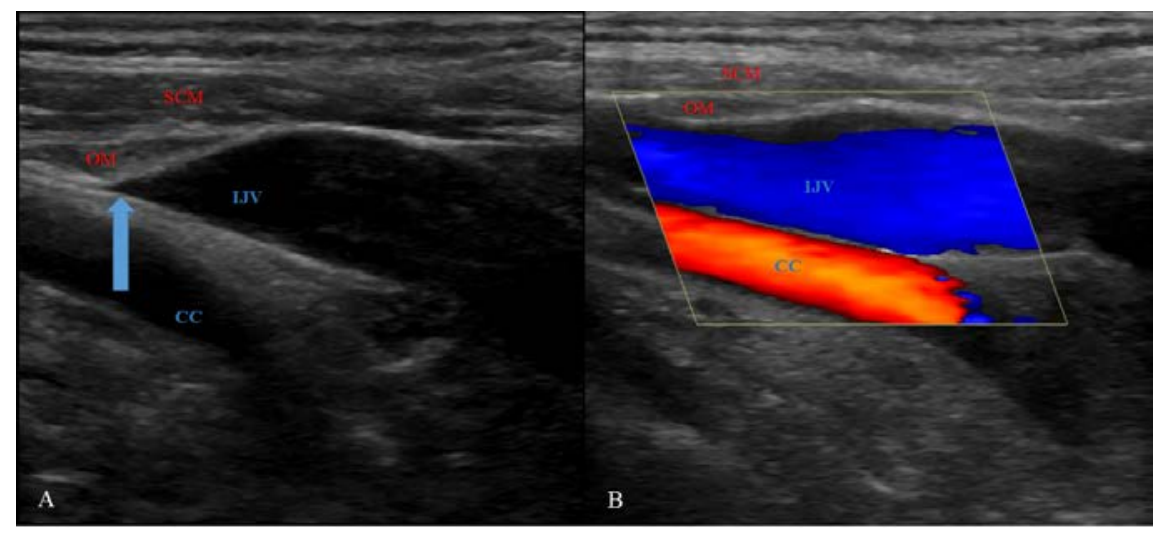

Figure 2. Case 2: ECD analysis of VV and IJVs (type 2 CCSVI, called Mechanical). A) complete muscular compression of left IJV in J2 and J3 segments (blue arrow) with no flow. B) significant basal reduction of muscle compression and a normalized outflow through IJVs after complete cycle of physiotherapy treatment for sternocleidomastoid and omohyoid muscles relaxation.
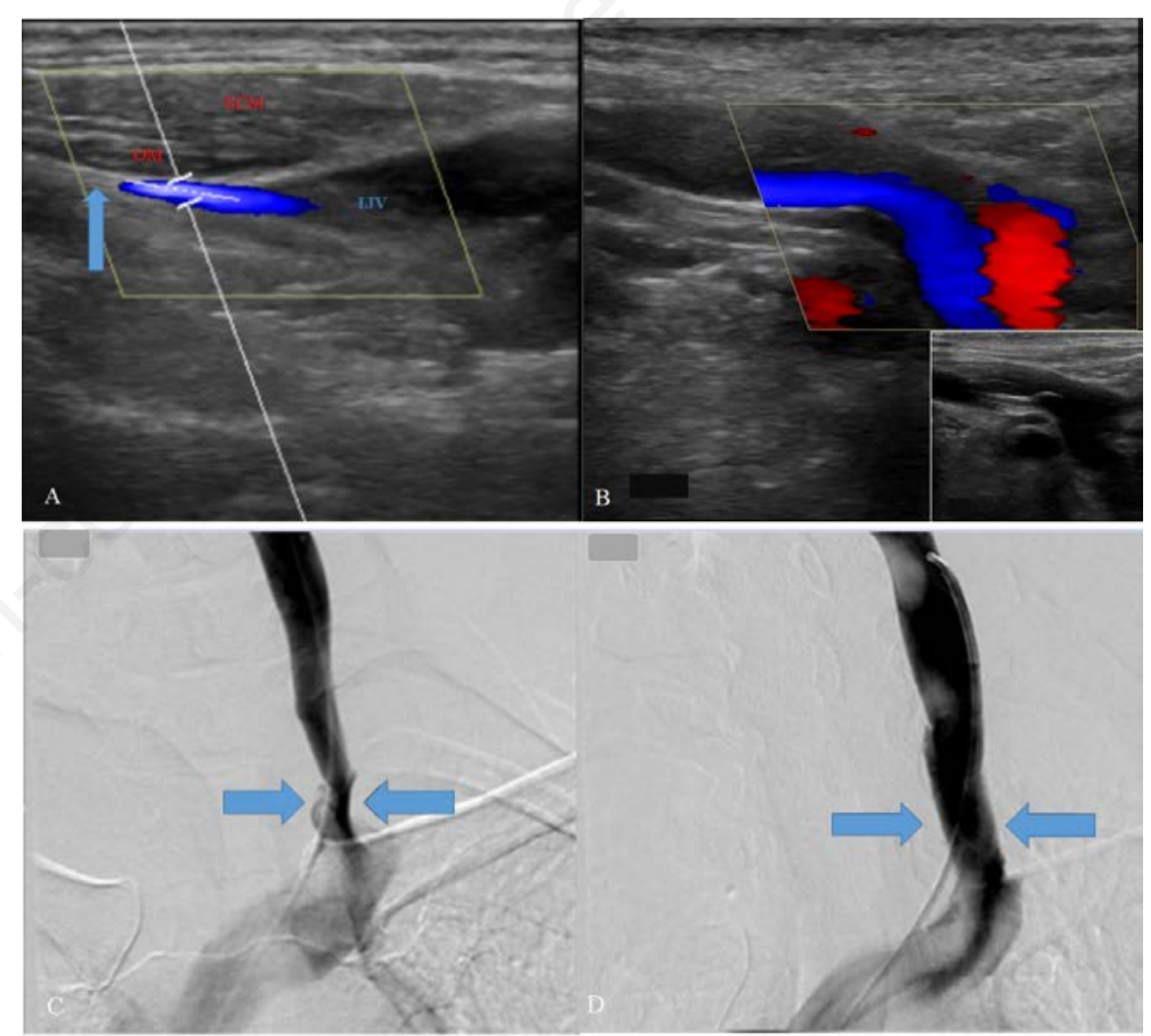

Figure 3. Case 3: ECD analysis of VV and IJVs (type 3 CCSVI, called Mixed). A) complete muscular compression of left IJV in $\mathrm{J} 2$ and $\mathrm{J} 3$ segments (blue arrow). B) immobile septum in $\mathrm{J1}$ segment determining annular constriction between $\mathrm{J} 1$ and succlavian vein (blue arrow) with reduction of venous caliber and flow acceleration (in the small down image the septum and constriction of J1 segment without Doppler flow); C) selective venography of left IJV after a complete cycle of physiotherapy treatment for sternocleidomastoid and omohyoid muscles relaxation, revealed a normalized vessel caliber in J2 and J3 segments, confirming the annular constriction between J1 segment and succlavian vein indicated by blue arrows. D) selective venography of left IJV after 14/40 mm balloon dilatation showing the resolution of vessel caliber constriction and normalized cerebral outflow (blue arrow). 
type 1 CCSVI due to an endovascular obstacle, called hydraulic, type 2 CCSVI due to a muscular compression without endovascular anomalies, called mechanical and type 3 CCSVI presenting both endovascular and extravascular anomalies, called mixed. We propose a flow chart which may allow (Figure 4) different treatment options according to the CCSVI patterns: selective venography of IJV and dilatation treatment in case of endovascular anomalies (type 1) as described before by our team ${ }^{11}$ and other hospital protocols as a safe and effective treatment in this form of anomalies ${ }^{12}$ and an adequate rehabilitation for SCM and OM muscles relaxation in case of mechanical extravascular obstruction (type 2) as described before by our team, first description of a conservative treatment for this anomaly as alternative to muscular resection $^{4}$ and a composed treatment in case of mixed pattern (type 3), according to ECD images and where feasible MR images (Table 1).

In fact, in CCSVI due to exclusively muscular entrapment of IJV (pattern 2) and/or in association with vascular anomalies (pattern 3), until recently literature described surgical resection of $\mathrm{OM}$ and SCM muscles as the only treatment option. ${ }^{13,14}$

IJV entrapment due to muscular compression was related not only to ear disorder as MéS, but recently De Bonis et al. have indicated the possible association with high-pressure hydrocephalus, and enlarged ventricles, calling this syndrome JEDI (jugular entrapment dilated ventricles intracranial hypertension). In this case, they describe as the jugular decompression has allowed a significant reduction of intracranial pressure. ${ }^{15}$

Zamboni and his team has showed significant data about pattern 3 CCSVI treatment, associating in the endophlebectomy treatment the removal of the defective valves with the muscle resection, achieving so an unique combined surgical treatment to solve both the anomalies (muscular and vascular) and allowing a restored cerebral ad ear outflow. ${ }^{16}$ Endophlebectomy is considered an alternative option to IJV angioplasty, when vascular anomalies (intraluminal defects as immobile valves, septum, inverted valve orientation) lend themselves to recidivism and muscular resection was considered the only possible muscular treatment, while recently our team has proposed with success a rehabilitative treatment to solve the muscle compression of IJV.

Here we describe the three different pattern of CCSVI, proposing the indication to follow for each pattern in terms of tailored treatment approach, as indicated in our flow chart (Figure 4). Surgical option represent the second choice in pattern 2 and 3 , if the conservative treatment is not effective. On the other hand, quite recently, different venographic patterns has been used to give tailored indication to venoplasty in case of CCSVI associated to multiple sclerosis. ${ }^{17}$

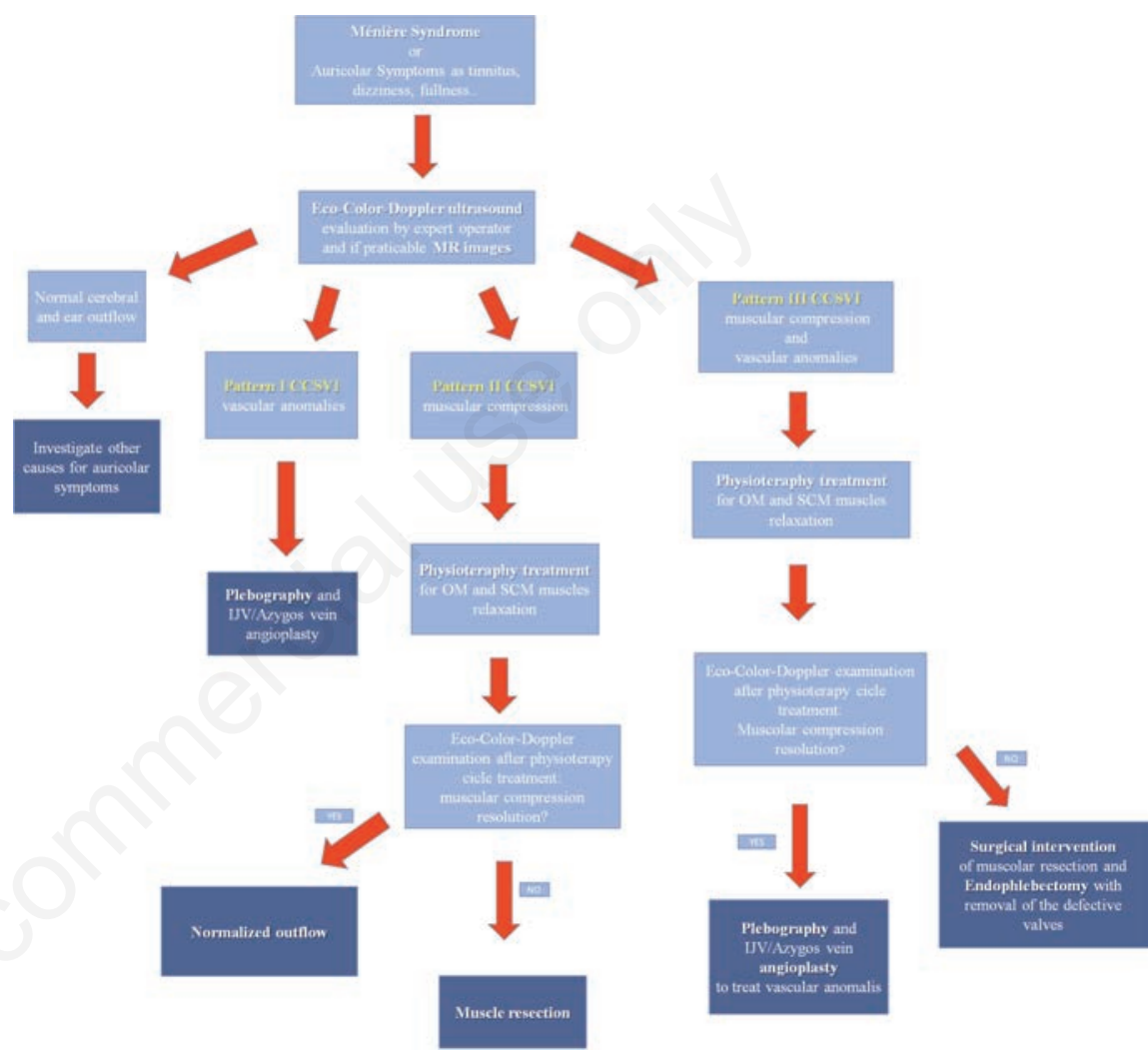

Figure 4. Flow chart for first describes different treatment options according to the CCSVI patterns.

Table 1. Description step by step of rehabilitative treatment.

Steps
$\begin{aligned} & \text { Description } \\ & \text { The patient is invited to position himself in clinostatism, to hyperextend the neck and to turn the head to the opposite side to the muscle to } \\ & \text { be treated (at first one side and then on the other one). We gently follow with the fingertips the anatomical position of the muscles starting } \\ & \text { from the mastoid process up to the insertion under the sternal or clavicular bar of the involved muscles along their entire length, with a } \\ & \text { sliding of them, pincer compressions, hyperextension, until to get a complete relaxation. After that, the patient is invited to position himself } \\ & \text { in ortostatism and we repeat the same treatment to obtain the same result in this position. } \\ & \text { Manual decontracting path is aimed at stretching every single muscle in the neck region. Particular attention must be paid to the } \\ & \text { sternocleidomastoid, the omohyoid, the styloid, the mylohyoid, infrahyoid, suprahyoid and digastrics muscles. The previous ECD analysis clearly } \\ & \text { gives information about the muscle involved in the IJV compression, the site (J1 and/or J2 and/or J3) and if the treatment should be bilateral or not }\end{aligned}$
$\begin{array}{ll}\text { Euch treatment lasts about } 60 \text { minutes for a total of } 12 / 15 \text { sessions. } \\ \text { Follow up }\end{array}$
$\begin{aligned} & \text { At the end of the established sessions, we evaluate symptoms, ECD analysis (to investigate the resolution or not of IJV muscle compression) and } \\ & \text { audiology examination, deciding if repeat again the treatment (if the results are not satisfactory) or recommending the patient a course of } \\ & \text { maintenance therapy every } 30 \text { days (if the results are satisfactory) in order to maintain the achieved benefits. }\end{aligned}$




\section{Conclusions}

Our flow chart allows to identify the three different patterns of CCSVI, by ECD images and (when it is feasible) RM images, giving indications about a tailored treatment approach for each one, proposing the innovation of rehabilitative treatment for muscular type (alone in pattern 2 or mixed with vascular anomalies in pattern $3)$. These treatment possibilities, in patients with a correct diagnosis of extracranial anomalous venous drainage, may represent a valid option in a scenario where conventional treatments are not very effective for this setting of patients.

\section{References}

1. Zamboni P, Morovic S, Menegatti E et al. Screening for chronic cerebrospinal venous in. sufficiency (CCSVI) using ultrasound - REchommendations for a protocol. Int Angiol 2011;30:571-97.

2. Lee AB, Laredo J, Neville R. Embryological background of truncular venous malformation in the extracranial venous pathways as the cause of chronic cerebro spinal venous insufficiency. Int Angiol 2010;29:95-108.

3. Mandolesi S, D'Alessandro A, Ciccone $\mathrm{MM}$, et al. Analysis of patients with chronic cerebro-spinal venous insufficiency and multiple sclerosis: identification of parameters of clinical severity. Veins and Lymphatics 2015;4:4570.

4. Piraino D, Garofalo G, Faletra A, Messina A. The omohyoid and sternocleidomastoid muscles entrapment of the internal jugular vein: Which role in
Mèniére disease patients? Treatment perspective description. Veins and Lymphatics 2018;7:7760.

5. Zamboni P. Why Current Doppler Ultrasound Methodology Is Inaccurate in Assessing Cerebral Venous Return: The Alternative of the Ultrasonic Jugular Venous Pulse. Behav Neurol 2016;7082856.

6. Toro EF, Borgioli F, Zhang Q, et al. Inner-ear circulation in humans is disrupted by extracranial venous outflow strictures: Implications for Ménière's disease. Veins and Lymphatics 2018;7:7156.

7. Zivadinov R, Bastianello S, Dake MD, et al. REchommendations for Multimodal Noninvasive and Invasive Screening for Detection of Extracranial Venous Abnormalities Indicative of Chronic Cerebrospinal Venous Insufficiency: A Position Statement of the International Society for Neurovascular Disease. J Vasc Interv Radiol 2014;25:1785-94.

8. Godlowski Z. Hyperosmosis of endolymph as primary pathogenic mechanism of Meniere's disease and its clinical management. Acta Otolaryngol Suppl 1972;299:1-36.

9. Alpini DC, Bavera PM, Di Berardino F, et al. Bridging the gap between chronic cerebrospinal venous insufficiency and Ménière disease. Veins and Lymphatics 2016;5:5687.

10. Bavera PM, Di Berardino F, Cecconi P, et al. Chronic cerebro-spinal insufficiency in multiple sclerosis and meniere disease: same background, different patterns? Veins and Lymphatics 2017;6:6533.

11. Piraino D, Andolina G, Messina A.
Truncular Venous Malformations in Ménière Disease Patients: The Key for a New Treatment Perspective? J Vasc Interv Radiol 2016;27:766-8.

12. Bruno A, Califano L, Mastrangelo D, et al. Chronic cerebrospinal venous insufficiency in Ménière's disease: diagnosis and treatment. Veins and Lymphatics 2014;3:3854.

13. Gianesini S, Menegatti E, Mascoli F, et al. The omohyoid muscle entrapment of the internal jugular vein. A still unclear pathogenetic mechanism. Phlebology 2014;29:632-5.

14. Simka M, Majewski E, Fortuna M, Zaniewski M. Internal jugular vein entrapment in a multiple sclerosis patient. Case Rep Surg 2012;2012: 293568.

15. De Bonis P, Menegatti E, Cavallo MA, et al. JEDI (jugular entrapment, dilated ventricles, intracranial hypertension) syndrome: a new clinical entity? A case report. Acta Neurochir (Wien) 2019; 161:1367-70.

16. Zamboni P, Menegatti E, Cittanti C, et al. Fixing the jugular flow reduces ventricle volume and improves brain perfusion. J Vasc Surg: Venous Lym Dis 2016;4:434-45.

17. Zamboni P, Galeotti R, Salvi F, et al.; Brave Dreams Research Group. Effects of Venous Angioplasty on Cerebral Lesions in Multiple Sclerosis: Expanded Analysis of the Brave Dreams Double-Blind, ShamControlled Randomized Trial. J Endovasc Ther 2019 Nov 17:15266028 19890110. doi: 10.1177/152660 2819890110. [Epub ahead oprint] Erratum in: J Endovasc Ther. 2020 Feb;27(1). 